\title{
Comparative study on functional outcome, complication and readmission rate in bilateral simultaneous versus staggered versus staged total knee replacement: a retrospective study
}

\author{
Vishal Ashokraj Pushkarna ${ }^{1 *}$, A. V. Gurava Reddy ${ }^{2}$
}

\begin{abstract}
${ }^{1}$ Department of Orthopaedics, Gujarat Adani Institute of Health Sciences, Bhuj, Gujarat, India
${ }^{2}$ Department of Joint Replacement Surgery, Sunshine Hospitals, Hyderabad, Telangana, India
\end{abstract}

Received: 31 March 2020

Revised: 07 June 2020

Accepted: 09 June 2020

\section{*Correspondence: \\ Dr. Vishal Ashokraj Pushkarna, \\ E-mail: vishalpusshkarna@hotmail.com}

Copyright: () the author(s), publisher and licensee Medip Academy. This is an open-access article distributed under the terms of the Creative Commons Attribution Non-Commercial License, which permits unrestricted non-commercial use, distribution, and reproduction in any medium, provided the original work is properly cited.

\begin{abstract}
Background: An important source of debate in orthopaedic practices is the choice of performing simultaneously, staggered or staged bilateral total knee arthroplasty (BTKA). Many studies are available which compare bilateral simultaneous vs staged TKA in terms of functional outcome. But still there are no studies which include staggered BTKA (done in a single hospital stay) and compared their functional outcome, associated complication with a 90 days readmission rate.

Methods: A retrospective review of 300 TKAs patients who underwent bilateral TKA. Patients were divided into three groups with 100 patients each in simultaneous, staggered, staged group. We use hospital electronic health records database to compare the complication and readmission rates in all three groups. Functional outcome was evaluated preoperatively using knee society score (KSS), Western Ontario and McMaster Universities Arthritis Index (WOMAC), Oxford knee score, Kujala score and range of motion.

Results: The study consists of 234 females and 66 males, among which maximum females were in a simultaneous group while male where more in staggered group. Mean age group in our study was $62.25 \%$. ASA score of 3 was significantly more in staged group. Patient in the staggered group had a better KSS and Kujala score with better range of motion and less readmission and complication rate. Simultaneous BTKA had slightly higher complication rate.

Conclusions: This study demonstrates that there is not big significant in functional outcome when comparing simultaneous, staged and staggered bilateral TKA, but still staggered group shows better postoperative functional outcome.
\end{abstract}

Keywords: Simultaneous, Staged, Staggered, BTKA

\section{INTRODUCTION}

Total knee arthroplasty (TKA) is mainly offered to patients with end-stage osteoarthritis (OA) and has become more prevalent in recent years. TKA is an effective intervention that improves quality of life, reduces pain and increases functional capability. ${ }^{1}$ Many patients in need of TKA have bilateral symptoms and require surgery for both extremities. Both patient and surgeon must decide whether to treat end-stage arthritis with simultaneous bilateral TKA or staggered TKA (both are done within same admission) or staged bilateral TKA with a certain time interval between the two procedures.

The advantages of having a simultaneous procedure include reduced cost, exposure to the risk of only one anesthetic, earlier return to baseline functionality and 
convenience for the patient. $^{1}$ However, having a simultaneous TKA causes a stress response and the response is directly related to the proportion of anesthetics. Previous studies have investigated differences in responses to staged bilateral and simultaneous bilateral TKA in terms of short-term discomfort, morbidity and mortality, and cost-effectiveness. There is no evidence-based guideline regarding the optimal choice between simultaneous vs staggered vs staged bilateral TKA. ${ }^{2}$ However, few studies have investigated differences in functional improvements. An Indian study described changes in functional improvements using the Western Ontario and McMaster University Osteoarthritis Index (WOMAC) in patients receiving simultaneous bilateral TKA, and another study from the United Kingdom reported changes in WOMAC scores in patients who received staged bilateral TKA. ${ }^{3}$ However, these studies lacked suitable controls, precluding group comparisons between studies. There is no literature comparing the functional outcome of bilateral simultaneous, staggered and staged TKR in one study with their complication and readmission rate in 90 days' time.

Thus, the objective of this study was to investigate differences in functional outcome, complication and readmission rate in patients receiving bilateral simultaneous vs staggered vs staged TKA.

\section{METHODS}

A retrospective review of 300 TKAs patients who underwent bilateral TKA in Sunshine Hospital between 2016 to 2017 were included. The patients were divided into three groups, those who underwent simultaneous bilateral TKA in one group, staggered bilateral TKA in one and staged bilateral TKA in one group.

Inclusion criteria were patients who underwent BTKA (simultaneous, staggered, staged). Exclusion criteria were patients who lost follow up and patient comes with complication or readmission beyond 3 months of primary surgery.

Patients were extracted from a surgical database encompassing surgery that were having at least two years follow up. The decision to proceed with simultaneous or staggered or staged procedure was made between the patient and surgeon based upon the patient's medical comorbidities and preference of the patient. Patient demographic data, ASA score, and preoperative medical comorbidity were documented from records. Functional outcome was evaluated pre operatively using knee society score (KSS), WOMAC, Oxford knee score (OKS) Kujala score and range of motion (ROM). ${ }^{3,4}$ Regarding complication rate and readmission data was obtained and analyzed from medical records of patients in our institute. All postoperative complication was categorized as either major or minor. ${ }^{5}$ Major complications included deep infection, cerebrovascular accident, myocardial infraction and pulmonary embolism and other complication were considered minor. Aseptic revision and septic revision cases were also considered.

Most of the statistical analysis was conducted using SPSS statistical software, whereas the post-operative complication rate was analyzed using chi-square test. For all analyses, a p value of 5 or less $(\mathrm{alpha}=0.05)$ was considered significant.

\section{RESULTS}

The study consists of 234 females $76.5 \%$ and 72 males $23.5 \%$, among which maximum females were in a simultaneous group $88 \%$ while male where more in staggered group $29 \%$. Mean age group in our study was $62.25 \%$. ASA score of 3 was significantly more in staged group $65 \%$ compared to the other two groups (Table 1).

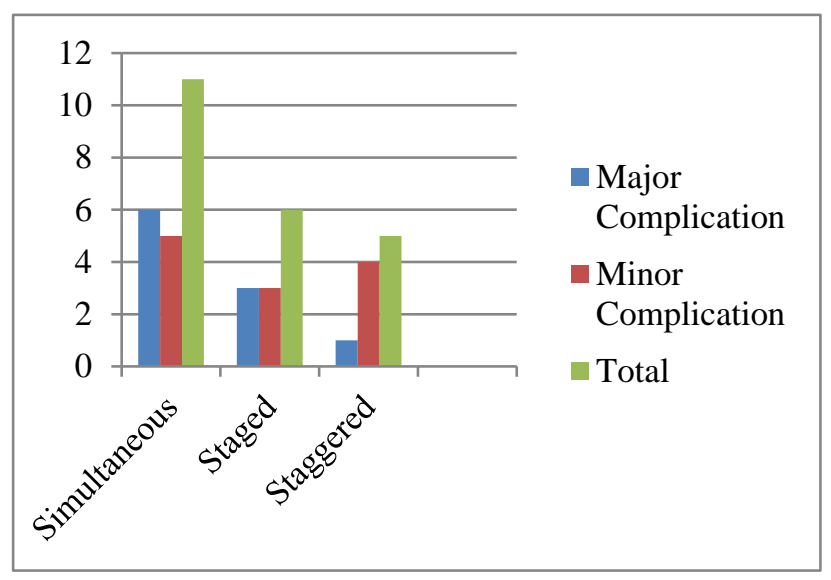

Figure 1: Complication in three groups.

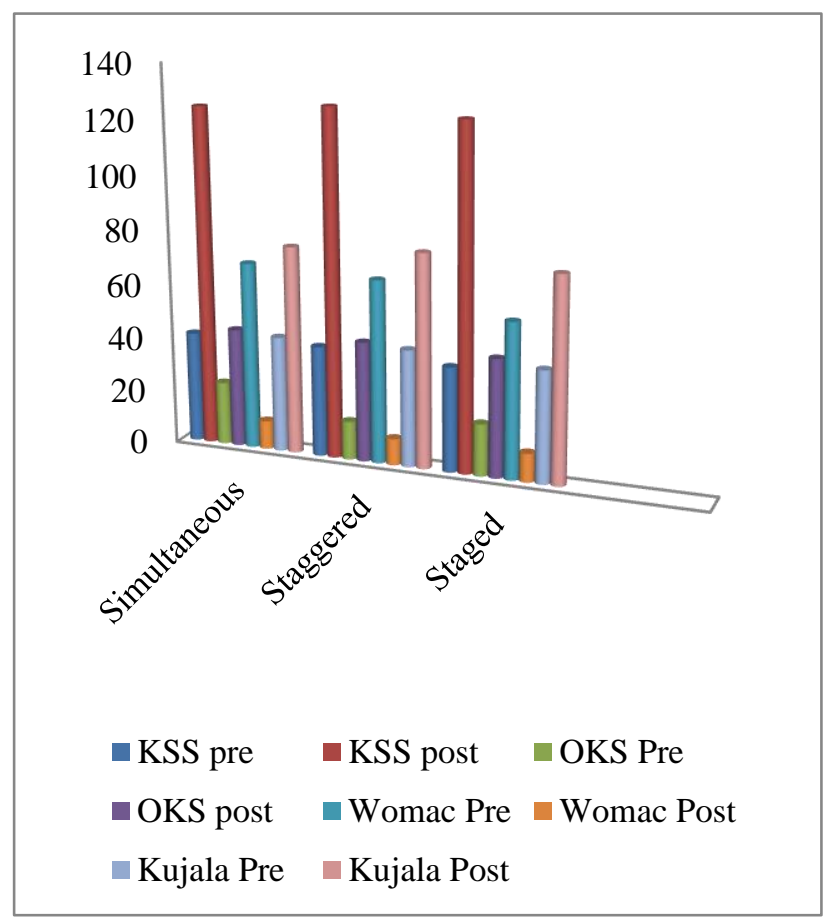

Figure 2: Distribution of patients as per score. 
Table 1: Demographic characteristics of patients used in this study.

\begin{tabular}{|llll|}
\hline Variables & Simultaneous $(\mathbf{n}=\mathbf{1 0 0})$ & Staggered $(\mathbf{n}=\mathbf{1 0 0})$ & Staged $(\mathbf{n}=\mathbf{1 0 0})$ \\
\hline Age & 60.86 & 62.69 & 63.20 \\
\hline Sex (male/female) & $16 / 84$ & $71 / 29$ & $73 / 27$ \\
\hline ASA class & 2.5 & 2.6 & 2.6 \\
\hline Follow-up period & 3.00 & 2.94 & 2.90 \\
\hline KSS & 40.79 & 40.81 & 38.65 \\
\hline WOMAC & 68.94 & 67.44 & 57.37 \\
\hline Kujala & 42.63 & 43.22 & 41.40 \\
\hline OKS & 23.13 & 14.16 & 19.25 \\
\hline
\end{tabular}

Table 2: Complication.

\begin{tabular}{|llll|}
\hline Complication & Simultaneous & Staggered & Staged \\
\hline Major complication & & & \\
\hline Myocardial infraction & 1 & 0 & 0 \\
\hline Pulmonary embolism & 0 & 0 & 0 \\
\hline Cerebrovascular accident & 1 & 0 & 3 \\
\hline Deep infection & 4 & 1 & 0 \\
\hline Minor complication & & 0 & 2 \\
\hline DVT & 1 & 2 & 1 \\
\hline Superficial infection & 2 & 2 & 6 \\
\hline UTI & 2 & 5 & \\
\hline Total & 11 & & \\
\hline Total complication rate & $7.3 \%$ & & \\
\hline
\end{tabular}

Table 3: Post-operative functional outcomes.

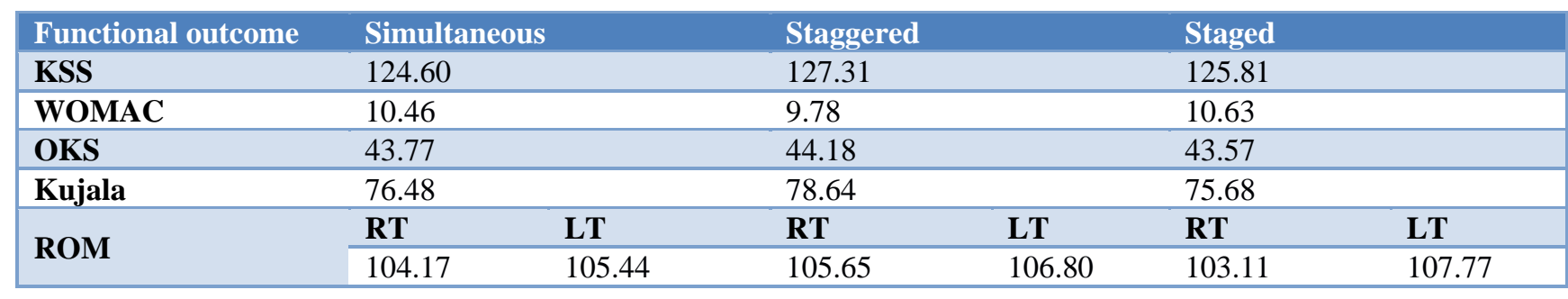

Patient in the staggered group had a better KSS score compared to patients with simultaneous and staged bilateral TKA group, but their $\mathrm{p}$ value was not that significant. WOMAC and OKS, there was no significant difference in all the three groups. Kujala score was better in staggered groups. Range of motion was better in staggered group specially in second knee compared to first one (Table 3) (Figure 2).

Table 4: Re-admission rate.

\begin{tabular}{|ll|}
\hline Variables & $\%$ \\
\hline Simultaneous & 9.0 \\
\hline Staggered & 5.0 \\
\hline Staged & 5.0 \\
\hline Total & 6.3 \\
\hline
\end{tabular}

The complication rate was higher in a simultaneous group with $11 \%$. Re-admission rate is higher in a simultaneous group with $9 \%$ compared to staggered and staged which was 5\% (Table 2-4) (Figure 1 and 3).

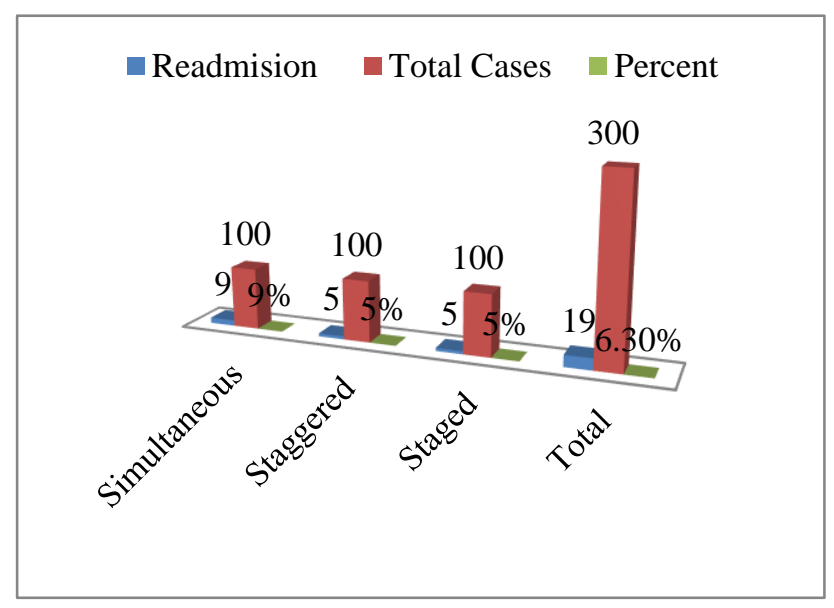

Figure 3: Re-admission rate. 


\section{DISCUSSION}

Knee arthritis is a common problem that may require treatment with TKR at its end stage. Today, TKR revolves around 3 principles: cost, complications, and outcome. With the single anesthesia administration and single hospital stay in simultaneous bilateral TKR result in reduced cost compared with unilateral TKR has been reported. The decision to go for simultaneous TKR or staggered or staged TKR depends on patients' needs and expectation and their physician's recommendation considering patient comorbidities. Simultaneous bilateral TKR has several advantages, there is still concern about the safety of this operation because it might be associated with a higher risk of perioperative complication. Despite these concerns, simultaneous bilateral TKA is routinely performed. Sometime due to comorbidities many patients undergo staged TKA or Staggered TKA in one hospital admission

The purpose of this study is to find benefits and risk associated with simultaneous, Staggered, Staged bilateral TKA. This study will focus on functional out, postoperative complication and readmission rate within 90 days of simultaneous, staggered and staged bilateral TKA. The result of our study suggests the overall complication rate was $2 \%$ with the simultaneous and staged group had similar rates of complication compared to less in staggered group. Readmission rate was higher in a simultaneous group which was around 9\% compared to staged which was around 5\% and staggered was around 5\%.

According to many authors, simultaneous bilateral TKA is associated with an increased risk of perioperative cardiac and pulmonary complications. ${ }^{2,3}$ Simultaneous bilateral TKA is reported.

To be associated with a 2 folds greater risk of cardiovascular complications than single unilateral TKA and a 1.6 folds greater risk compared to staged bilateral TKA. $^{2,4}$ However, several series have reported that simultaneous bilateral TKA is safer. One of the earliest series of Hardaker et al compared the perioperative safety of simultaneous bilateral TKA and that of staged TKA. ${ }^{5}$ They found no differences in complication rates or outcomes. Morrey et al. $^{6}$ retrospectively studied 290 simultaneous bilateral TKAs and compared them with staged bilateral TKAs. They found similar rates of complications and mortality between the two groups. Recent studies continue to demonstrate increased complications from bilateral TKAs under the same anesthetics, whereas others show comparable safety profiles with good outcomes. ${ }^{7-9}$ Kim et al found no significant difference in the overall number of complications or perioperative mortality in a simultaneous bilateral TKA group compared to the unilateral TKA group. ${ }^{10}$ The limitations of this study include a retrospective design and the use of observable data. The results of the present study must be interpreted with great caution due to its non-randomized retrospective design. In addition, patient selection criteria remain an issue of concern for simultaneous bilateral TKA. If we want the appropriate statistical power analysis, this study needs more cases.

\section{CONCLUSION}

This study demonstrates that there is not big significant in functional outcome when comparing simultaneous, staged and staggered bilateral TKA, but still staggered group shows better postoperative functional outcome compared with first knee second knee shows better outcome. Our results show that there is slightly increased in major complication in simultaneous bilateral TKA compared with staged and staggered groups. There is still a lack of evidence to support the superiority of either BTKA simultaneous or staged or staggered. Still simultaneous bilateral TKA remains a safe option for appropriately motivated patient and lower risk patient.

Funding: No funding sources Conflict of interest: None declared

Ethical approval: The study was approved by the Sunshine Hospital Smart Ethical Committee

\section{REFERENCES}

1. Ritter MA, Harty LD, Davis KE, Meding JB, Berend M. Simultaneous bilateral, staged bilateral, and unilateral total knee arthroplasty: a survival analysis. J Bone Joint Surg Am. 2003;85(8):1532-7.

2. Restrepo C, Parvizi J, Dietrich T, Einhorn TA. Safety of simultaneous bilateral total knee arthroplasty: a meta-analysis. J Bone Joint Surg Am. 2007;89(6):1220-6.

3. Memtsoudis SG, Hargett M, Russell LA, Parvizi J, Baril CWL, Stundner O, et al. Consensus statement from the consensus conference on bilateral total knee arthroplasty group. Clin Orthop Relat Res. 2013;471(8):2649-57.

4. Meehan JP, Danielsen B, Tancredi DJ, Kim S, Jamali AA, White RH. A population-based comparison of the incidence of adverse outcomes after simultaneous-bilateral and staged-bilateral total knee arthroplasty. J Bone Joint Surg Am. 2011;93(23):2203-13.

5. Hardaker WT, Ogden WS, Musgrave RE, Goldner JL. Simultaneous and staged bilateral total knee arthroplasty. J Bone Joint Surg Am. 1978;60(2):24750.

6. Morrey BF, Adams RA, Ilstrup DM, Bryan RS. Complications and mortality associated with bilateral or unilateral total knee arthroplasty. J Bone Joint Surg Am. 1987;69(4):484-8.

7. Yoon HS, Han CD, Yang IH. Comparison of simultaneous bilateral and staged bilateral total knee arthroplasty in terms of perioperative complications. J Arthroplasty. 2010;25(2):179-85.

8. Oakes DA, Hanssen AD. Bilateral total knee replacement using the same anaesthetic is not 
justified by assessment of the risks. Clin Orthop Relat Res. 2004;428:87-91.

9. Shin YH, Kim MH, Ko JS, Park JA. The safety of simultaneous bilateral versus unilateral total knee arthroplasty: the experience in a Korean hospital. Singapore Med J. 2010;51(1):44-9.

10. Kim YH, Choi YW, Kim JS. Simultaneous bilateral sequential total knee replacement is as safe as unilateral total knee replacement. J Bone Joint Surg Br. 2009;91(1):64-8.
11. Gabr A, Withers D, Pope J, Santini A. Functional outcome of staged bilateral knee replacements. Ann R Coll Surg Engl. 2011;93(7):537.

Cite this article as: Pushkarna VA, Reddy AVG. Comparative study on functional outcome, complication and readmission rate in bilateral simultaneous versus staggered versus staged total knee replacement: a retrospective study. Int J Res Orthop 2020;6:783-7. 